\title{
Prenatal and postnatal exposure to ambient air pollution and asthma in neonatal jaundice infants
}

\author{
Hao-Wei Chung ${ }^{1}$, Chih-Hsing Hung ${ }^{2}$, Fu-Chen Kuo ${ }^{3}$, Hui-Min Hsieh ${ }^{4}$, Chung-Hsiang Li $^{5}$, \\ Yi-Ching $\operatorname{Lin}^{5}$, Yu-Hsiang Tsao ${ }^{4}$, and Huang-Wei $\mathrm{Wu}^{1}$ \\ ${ }^{1}$ Kaohsiung Municipal Hsiao-kang Hospital \\ ${ }^{2}$ Kaohsiung Medical University College of Medicine \\ ${ }^{3}$ E-Da Hospital \\ ${ }^{4}$ Kaohsiung Medical University \\ ${ }^{5}$ Kaohsiung Medical University Chung Ho Memorial Hospital
}

January 3, 2022

\begin{abstract}
Background: Both air pollutants and neonatal jaundice (NJ) have known effects on childhood asthma, but higher total serum bilirubin (TSB) level was associated with lung protection. This study aimed to assess whether prenatal/postnatal exposure to air ambient pollutants is related to the inception of asthma in NJ infants. Material and methods: A nested case-control retrospective study of NJ infants was performed on the Kaohsiung Medical University Hospital Research Database between 2009 and 2019. The average concentration of particulate matter (PM2.5), sulfur dioxide (SO2), nitric dioxide (NO2) for six months, first and second years after the birth, and first, second and third trimesters prenatally were analyzed. The mild and significant NJ infants were categorized as TSB level < and [?]12 mg/dl, respectively. Asthma was defined as a diagnosis with medication. The adjusted odds ratio (aOR) and 95\% confidence interval (CI) present the relationship between study periods and childhood asthma. Results: SO2 and NO2 exposure during prenatal periods were significantly associated with increased risk of childhood asthma in mild NJ infants (aOR (95\% CI)), SO2: 1.20-1.34 (1.05-1.56); NO2: 1.06-1.07 (1.01-1.13)). Effects were more pronounced in postnatal exposure to three ambient air pollutants in mild jaundice infants. (aOR (95\% CI), SO2: 1.33-1.41 (1.14-1.69); NO2: 1.07-1.31 (1.01-1.49; PM2.5:1.05 (1.00-1.10) Conclusion: Both SO2 and NO2 during prenatal and postnatal exposure in mild NJ infants were associated with childhood asthma. Whether taken phototherapy or not, significant NJ infants were spared by three ambient air pollutants.
\end{abstract}

\section{Hosted file}

prenatl and postanatl amibient air in jaundice infants main feature 1225final.doc available at https://authorea.com/users/453609/articles/551409-prenatal-and-postnatal-exposure-toambient-air-pollution-and-asthma-in-neonatal-jaundice-infants

\section{Hosted file}

fiqrue 1.docx available at https://authorea.com/users/453609/articles/551409-prenatal-andpostnatal-exposure-to-ambient-air-pollution-and-asthma-in-neonatal-jaundice-infants 

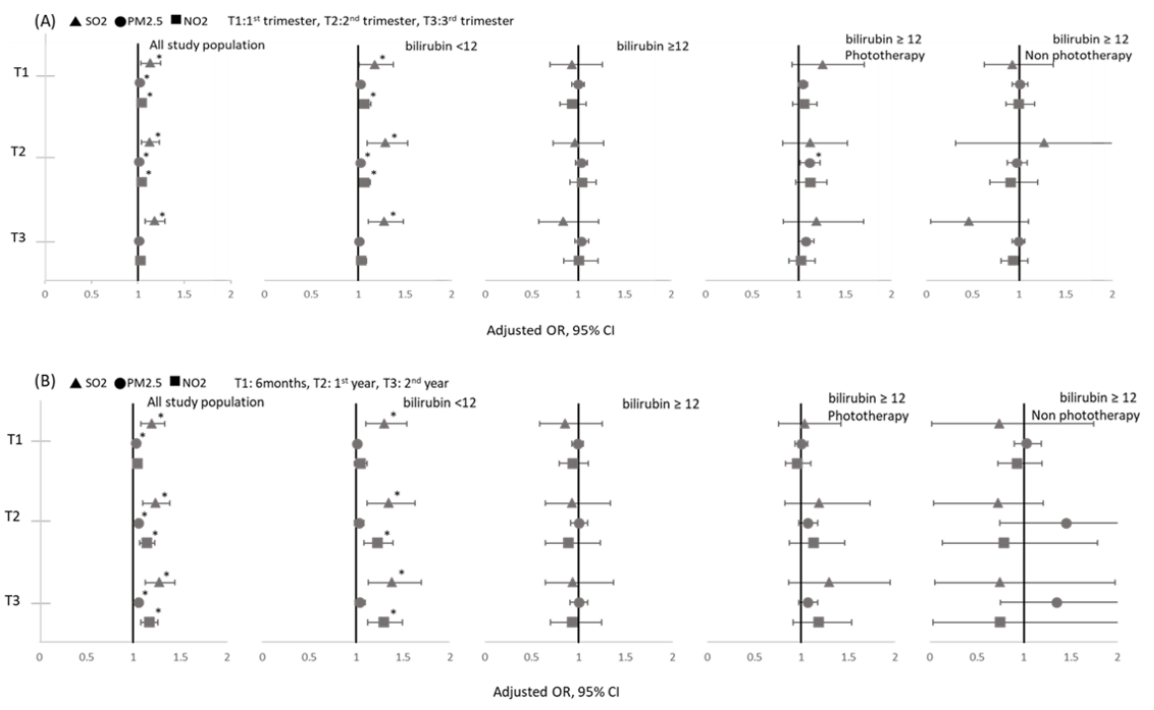